\title{
Evaluation of different respiratory samples and saliva for the detection of SARS CoV-2 RNA
}

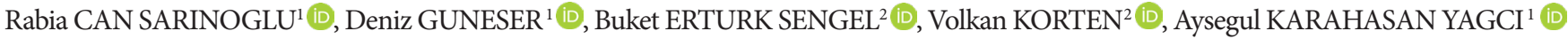

\begin{abstract}
${ }^{1}$ Department of Medical Microbiology, School of Medicine, Marmara University, Pendik Training and Research Hospital, Istanbul, Turkey. ${ }^{2}$ Department of Infectious Diseases and Clinical Microbiology, School of Medicine, Marmara University, Pendik Training and Research Hospital, Istanbul, Turkey.
\end{abstract}

Corresponding Author: Rabia CAN SARINOGLU

E-mail: rabiacansarinoglu@hotmail.com

Submitted: $13.10 .2020 \quad$ Accepted: 10.12.2020

\begin{abstract}
Objective: We aimed to analyse the positivity rate and cycle threshold values indicating viral loads for SARS CoV-2 among different respiratory specimens and also to evaluate the diagnostic efficacy of saliva samples.

Materials and Methods: We included combined oropharyngeal and nasopharyngeal swab (cONS), sputum, and tracheal aspirate (TA) specimens of patients. Unpreserved saliva samples were collected prospectively from hospitalized patients within 72 hours of admission. SARS CoV-2 RNA was extracted by using Bio-Speedy viral nucleic acid buffer than RT-PCR was performed with BioSpeedy COVID-19 qPCR detection kit.

Results: Retrospective evaluation revealed SARS CoV-2 RNA in 19.66\% of cONS (n: 5819), 30.77\% of sputum (n: 39 ), 29.41\% of TA samples (n: 34) from 4812 patients. In the majority (86.72\%) of the samples, the first cONS sample was positive. Consecutive cONS and sputum/TA samples were investigated in 52 patients of whom 11 were positive with either of these samples. Saliva positivity was detected in $60 \%$ of cONS positive (n: 20 ) and $30 \%$ of cONS negative (n: 12 ) patients.

Conclusion: Although, cONS samples show the greatest diagnostic guidance, repeated sampling from multiple sites of the respiratory tract increases the possibility of COVID-19 diagnosis. Saliva samples might be considered as an alternative specimen.

Keywords: SARS CoV-2, COVID-19, RT-PCR, Upper respiratory samples, Saliva, Cycle threshold
\end{abstract}

\section{INTRODUCTION}

In December 2019, a new coronavirus was identified in a group of patients in Wuhan, China [1]. Originally named as 2019 new coronavirus (novel Coronavirus, 2019-nCoV), the virus is currently named as severe acute respiratory syndrome coronavirus 2 (SARS CoV-2) by the International Committee on Taxonomy of Viruses (ICTV), and the disease caused by this virus is named as coronavirus disease 2019 (COVID-19) [2].

Since, the World Health Organization (WHO) declared the outbreak as a Public Health Emergency of International concern on January 30, 2020; COVID-19 affected 63, 719, 213 cases and 1, 482, 084 deaths were recorded by December 3, 2020 [3]. The first case was detected on March 10, 2020 in Turkey, 513, 656 cases and 14, 129 deaths have been declared by the Turkish Ministry of Health, on December 2, 2020 [4].

The current diagnostic method is the detection of SARS CoV-2 RNA by reverse transcriptase polymerase chain reaction (RTPCR) from clinical materials. The nasal swab (NS) refers to flocked swab stick sampling of the anterior nasal cavity and the nasopharyngeal swab (NPS) involves the introduction of a flocked swab stick deep into the nasopharynx (beyond the hardsoft palate transition) to achieve direct contact with the posterior nasopharyngeal mucosal wall [5]. Zou et al., compared 72 NS/ NPS specimens across various days of illness in 18 COVID-19 patients and concluded that NPS technique is time-consuming, resource intensive, and unsuitable for mass testing in a pandemic situation [6]. Although, sampling by oropharyngeal swab (OPS) is easier, it has a low negative predictive value. Xie et al., reported that only 9 out of 19 (47\%) OPS from ultimately seropositive COVID-19 patients were positive, calling attention to the importance of repeated sampling from multiple sites, including the lower respiratory system, in order to increase the diagnostic yield [7]. At present, the United States Centers for Disease Control and Prevention also recommends that nasopharyngeal and oropharyngeal flocked swabs should be

How to cite this article: Can Sarinoglu R, Guneser D, Erturk Sengel B, Korten V, Karahasan Yagci A. Evaluation of different respiratory samples and saliva for the detection of SARS-CoV-2 RNA. Marmara Med J 2021;34:(1):51-56. doi: 10.5472/marumj.866658 
used for the collection of specimens for SARS CoV-2 viral detection. While these methods have their limitations, both allow rapid up-scaling for mass testing and detection, as part of most countries' greater strategy to proactively test, isolate and contact-trace infected cases [8]. Turkish Ministry of Health diagnostic guideline advises using combined oropharyngeal and nasopharyngeal swab (cONS) samples from clinically suspected cases for diagnosis [9]. Lower respiratory sampling methods lack rapid up-scaling potential but the superiority of sputum for SARS CoV-2 viral detection was echoed by Pan et al., who demonstrated that sputum samples generally showed higher viral loads than throat swabs [10]. Tracheal aspirates (TA) may be obtained via suction from an indwelling endotracheal tube in mechanically ventilated patients, or direct tracheal suction of tracheotomized patients. Comparisons of upper respiratory samples vs. endotracheal aspirates in a cohort of 16 intubated COVID-19 patients showed that the latter had significantly higher viral RNA values compared to NS and OPS samples [11].

Recently, alternative strategies for specimen collection, including home collection by patients themselves have also been considered [12]. Testing saliva as an alternative first-line screening test especially in the face of shortages of swabs and personal protective equipment is advised [13]. To et al., reported that 20 of 23 (87\%) patients who had SARS CoV-2 detected by RT-PCR in NPS or sputum also had SARS CoV-2 detectable in saliva [14].

Herein, we shared our experience of COVID-19 laboratory diagnosis during the pandemic. We analyzed the positivity rate among upper and lower respiratory tract specimens, compared viral load indicated by cycle threshold $(\mathrm{Ct})$ values of RT-PCR assays, and evaluated the diagnostic efficacy of saliva samples.

\section{MATERIALS and METHODS}

We examined cONS, sputum and TA specimens collected from COVID-19 suspected patients admitted to Marmara University Pendik Training and Research Hospital between March 23 and September 15, 2020. Although, it is not included in our routine diagnostic procedures, we also evaluated saliva samples prospectively from a small group of hospitalized patients within 72 hours of admission.

cONS samples were taken from patients with acute respiratory illness (fever and at least one sign/symptom of respiratory disease, e.g., cough, shortness of breath) with either a history of travel to a location reporting community transmission of COVID-19 disease or having been in close contact with a confirmed COVID-19 case in the last 14 days before symptom onset; or a patient with severe acute respiratory illness requiring hospitalization without an alternative diagnosis that fully explains the clinical presentation. Sputum samples were obtained from patients with productive cough and TA specimens were taken from mechanically ventilated patients. cONS samples were taken into a transfer tube containing viral transport medium whereas sputum samples and TA samples were put into a $25 \mathrm{ml}$ sterile cup without viral transport media (VTM).

To obtain unpreserved saliva samples, patients were asked not to have any food or drink 30 minutes before collection, to pool saliva in their mouth for 1-2 minutes, and spit 1-2 mL of saliva into a sterile cup without VTM. Patient selection was done randomly regardless of severity of the infection. All samples were transferred to the laboratory within a basic triple packing system [15].

Viral RNA was extracted by using Bio-speedy ${ }^{\circledR}$ viral nucleic acid buffer (Bioexen LTD, Istanbul, Turkey) and RT-PCR was performed with Bio-speedy ${ }^{\circledast}$ COVID-19 qPCR detection kit, Version 2 (Bioexen LTD, Istanbul, Turkey) using primers and probes targeting the RNA-dependent RNA polymerase (RdRp) gene fragment in a LightCycler 96 System (Roche, Switzerland). Each $20 \mu \mathrm{L}$ reaction mixture contained $5 \mu \mathrm{L}$ of Oligo Mix, $10 \mu \mathrm{L}$ of $2 \mathrm{X}$ Prime Script Mix, and $5 \mu \mathrm{L}$ of RNA as the template. The thermal cycling condition was 15 minutes at $45^{\circ} \mathrm{C}$ for reverse transcription, 3 minutes at $95^{\circ} \mathrm{C}$ for PCR initial activation, and 45 cycles of 5 seconds at $95^{\circ} \mathrm{C}$ and 35 seconds at $55^{\circ} \mathrm{C}$ according to the manufacturer's instructions (Bioexen LTD, Istanbul, Turkey). Oligo Mix contains internal control (IC) targeting the Human RNase P gene as an extraction control. A positive and negative control were included in each run to generate a valid result. A Ct value of less than 40 was defined as a positive result. Analytical and clinical performance of the kit was determined by the "Turkish Ministry of Health, General Directorate of Public Health, Department of Microbiology Reference Laboratories and Biological Products (HSGM)". The analytical sensitivity of the kit is $99.4 \%$ and its specificity is $99.0 \%$.

The study protocol was approved by both the Turkish Ministry of Health (Protocol Number:2020-05-05T12_38_13) and the Institutional Review Board and Ethics Committee of Marmara University, Faculty of Medicine (Protocol Number: 09.2020.620).

\section{Statistical Analysis}

Statistical analyses were performed by using SPSS version 21.0 (SPSS Inc., Chicago, IL, USA). Descriptive statistics were presented as percentages and medians (IQR) in data without normal distribution. We used the Mann-Whitney $U$ test to compare continuous variables for independent groups. All tests were two-tailed; $\mathrm{P}$ values of $<0.05$ were considered to indicate statistical significance.

\section{RESULTS}

Among 4812 suspected COVID-19 patients, 1053 cases (532 male, 521 female) were confirmed positive by RT-PCR assay. The median age was 40 years (IQR:0-95). The most common symptom was cough $(66.22 \%)$, followed by dyspnea $(31.69 \%)$ and fever $(31.66 \%)$. The majority of the patients (\%59.79) had been in close contact with a positive patient in the last 14 days before symptom onset (Table I). 
Table I. Characteristics of patients with confirmed diagnosis of COVID-19 (n: 1053)

\begin{tabular}{lcc}
\hline Gender & $\mathbf{n}$ & $\%$ \\
\hline Male & 532 & 50.50 \\
Female & 521 & 49.50 \\
\hline Age, years & Median & IQR \\
\hline Male & 40 & $0-90$ \\
Female & 38 & $0-95$ \\
Total & 40 & $0-95$ \\
\hline Presenting signs and symptoms & $\mathbf{n}$ & $\%$ \\
\hline Cough & 697 & 66.22 \\
\hline Dyspnea & 334 & 31.69 \\
\hline Fever & 333 & 31.66 \\
\hline Epidemiological characteristics & $\mathbf{n}$ & $\%$ \\
\hline Contact with positive patient & 519 & 59.79 \\
\hline Travel history & 7 & 0.91 \\
\hline
\end{tabular}

SARS CoV-2 RNA has been investigated in cONS samples ( $\mathrm{n}$ : 5819), sputum (n:39), and TA (n: 34) samples from 4812 patients with suspected COVID-19 infection. Positivity was detected in $19.66 \%$ of cONS, $29.41 \%$ of TA, and $30.77 \%$ of sputum samples. Ct values of cONS samples were lower than sputum and TA samples, indicating higher viral loads (Table II).

Table II. Detection rates of SARS CoV-2 RNA from different respiratory tract samples. (cONS: combined oropharyngeal and nasopharyngeal swab, TA: tracheal aspirate)

\begin{tabular}{lcccc}
\hline & \multicolumn{3}{c}{ Sample Type (n) } & \\
& cONS (5819) & Sputum (39) & TA (34) & $\begin{array}{c}\text { TOTAL } \\
(5892)\end{array}$ \\
\hline $\begin{array}{l}\text { Positive test } \\
\text { result, n (\%) }\end{array}$ & $1144(19.66)$ & $12(30.77)$ & $10(29.41)$ & $1166(19.79)$ \\
$\begin{array}{l}\text { Cycle threshold, } \\
\text { median (IQR) }\end{array}$ & $27.00(14-40)$ & $30.05(21-39)$ & $30.00(24-39)$ & $27.17(14-40)$ \\
\hline
\end{tabular}

The results of repetitive cONS samples collected within 72 hours were evaluated retrospectively. In the majority of the patients (86.72\%) SARS CoV-2 RNA was detected in the first collected cONS sample. Out of 689 patients with initial negative results, the second test was performed. For these patients, the test results were positive in 98 cases (9.36\%). Thirty-nine patients $(3.72 \%)$ were found to have a positive RT-PCR result after two consecutive negative results. Two patients were tested positive by four repeated RT-PCR tests. Totally 139 patients (13.28\%) were diagnosed with COVID-19 through repetitive samples taken within maximum nine days (Table III).

Table III. Positivity rate of the repetitive cONS samples from COVID-19 suspected patients

\begin{tabular}{lccc} 
& Negative & \multicolumn{2}{c}{ Positive } \\
& $\mathrm{n}$ : $^{\text {a }}$ & $\mathrm{n}: 1047$ & $\%$ \\
\cline { 2 - 4 } 1. Sample & 4792 & 908 & 86.72 \\
b2. Sample & 591 & 98 & 9.36 \\
b3. Sample & 114 & 39 & 3.72 \\
b4. Sample & 15 & 2 & 0.19 \\
\hline
\end{tabular}

${ }^{a}$ since there are overlapping negative results obtained from the same patients, total number of negative samples were not added, ${ }^{b}$ repetitive cONS samples were collected within 72 hours. The maximum number of days of sample collection $\left(1^{\text {st }}-4^{\text {th }}\right)$ was nine.
Consecutive cONS and sputum or TA samples from 52 patients were investigated for the presence of SARS CoV-2 RNA. Eleven of 52 patients were found to be positive with either of these samples (Table IV). Detection time for SARS CoV-2 RNA in lower respiratory tract specimens ( 8 sputum, 3 TA) after the first clinical symptom varied 3 to 12 days. The difference in time between cONS and lower respiratory tract samples collection varied from 1 to 10 days. Whereas, in only four patients both cONS and sputum samples (patients 1-4) and in only one patient both cONS and TA samples (patient 5) were positive. The median Ct value was 30.00 (IQR: 25.85-39.92) in sputum and TA samples and 25.00 (IQR: 18.01-26.39) in cONS samples. Cycle threshold values detected from cONS samples were significantly lower than sputum and TA samples $(\mathrm{p}<0.05)$. Seven patients' (patient no: 2-6 and 10,11$)$ clinical condition was severe, requiring oxygen support or admission to the intensive care unit (Table V).

Table IV. Comparison of SARS CoV-2 RT-PCR results in consecutive cONS and lower respiratory tract samples (sputum and tracheal aspirate) $(n=52)$

\begin{tabular}{llccc} 
& & \multicolumn{4}{c}{ Sputum/TA Samples } \\
\cline { 3 - 5 } & & Positive n (\%) & Negative n (\%) & Total n (\%) \\
\hline \multirow{2}{*}{ cONS } & Positive n (\%) & $5(71.43)$ & $2(28.57)$ & $7(100.0)$ \\
\multirow{2}{*}{ Samples } & Negative n (\%) & $6(13.33)$ & $39(86.67)$ & $45(100.0)$ \\
& Total n (\%) & $11(21.15)$ & $41(78.85)$ & $52(100.0)$ \\
\hline \multicolumn{2}{l}{ cONS: combined oropharyngeal and nasopharyngeal swab, TA: tracheal aspirate }
\end{tabular}

Table V. Comparison of consecutive cONS and SARS CoV-2 RNA detected sputum/TA samples

\begin{tabular}{|c|c|c|c|c|}
\hline No & Specimen type & Result & Ct value ${ }^{*}$ & Days from symptom onset to test \\
\hline \multirow[t]{2}{*}{1} & cONS & $\mathrm{P}$ & 22.98 & 4 \\
\hline & Sputum & P & 28.25 & 6 \\
\hline \multirow[t]{3}{*}{$2^{*}$} & cONS & $\mathrm{P}$ & 18.01 & 2 \\
\hline & cONS & $\mathrm{N}$ & & 11 \\
\hline & Sputum & $\mathrm{P}$ & 32.90 & 12 \\
\hline \multirow[t]{2}{*}{$3^{*}$} & cONS & $\mathrm{P}$ & 22.27 & 2 \\
\hline & Sputum & $\mathrm{P}$ & 31.43 & 10 \\
\hline \multirow[t]{2}{*}{$4^{*}$} & cONS & P & 26.39 & 0 \\
\hline & Sputum & $\mathrm{P}$ & 30.93 & 5 \\
\hline \multirow[t]{2}{*}{$5^{*}$} & cONS & $\mathrm{P}$ & 26.04 & 3 \\
\hline & TA & $\mathrm{P}$ & 30.40 & 4 \\
\hline \multirow[t]{2}{*}{$6^{*}$} & cONS & $\mathrm{N}$ & & 2 \\
\hline & Sputum & $\mathrm{P}$ & 29.46 & 6 \\
\hline \multirow[t]{2}{*}{7} & cONS & $\mathrm{N}$ & & 1 \\
\hline & Sputum & $\mathrm{P}$ & 39.92 & 3 \\
\hline \multirow[t]{2}{*}{8} & cONS & $\mathrm{N}$ & & 2 \\
\hline & Sputum & $\mathrm{P}$ & 36.19 & 3 \\
\hline \multirow[t]{2}{*}{9} & cONS & $\mathrm{N}$ & & 0 \\
\hline & Sputum & $\mathrm{P}$ & 25.85 & 3 \\
\hline \multirow[t]{2}{*}{$10^{*}$} & cONS & $\mathrm{N}$ & & 0 \\
\hline & TA & $\mathrm{P}$ & 27.97 & 4 \\
\hline \multirow[t]{2}{*}{$11^{*}$} & cONS & $\mathrm{N}$ & & 5 \\
\hline & TA & P & 34.33 & 12 \\
\hline
\end{tabular}

"clinical condition was severe, hypoxic and/or intensive care unit admission, cONS: combined oropharyngeal and nasopharyngeal swab, N: negative, P: positive, TA: tracheal aspirate, ${ }^{*} p<0.05$. 
Table VI. Comparison of cONS and saliva samples

\begin{tabular}{|c|c|c|c|c|}
\hline No & Specimen type & Result & Ct value ${ }^{*}$ & $\begin{array}{c}\text { Days from symptom } \\
\text { onset to test }\end{array}$ \\
\hline \multirow{2}{*}{1} & cONS & $\mathrm{P}$ & 25.42 & 7 \\
\hline & Saliva & $\mathrm{N}$ & & 8 \\
\hline \multirow{2}{*}{2} & cONS & $\mathrm{P}$ & 21.95 & 5 \\
\hline & Saliva & $\mathrm{P}$ & 27.79 & 8 \\
\hline \multirow{2}{*}{3} & cONS & $\mathrm{P}$ & 23.20 & 0 \\
\hline & Saliva & $\mathrm{P}$ & 31.00 & 2 \\
\hline \multirow{2}{*}{4} & cONS & $\mathrm{P}$ & 27.59 & 0 \\
\hline & Saliva & $\mathrm{N}$ & & 1 \\
\hline \multirow{2}{*}{5} & cONS & $\mathrm{P}$ & 22.12 & 9 \\
\hline & Saliva & $\mathrm{N}$ & & 12 \\
\hline \multirow{2}{*}{6} & cONS & $\mathrm{P}$ & 26.03 & 4 \\
\hline & Saliva & $\mathrm{P}$ & 30.80 & 6 \\
\hline \multirow{2}{*}{7} & cONS & $\mathrm{P}$ & 23.56 & 2 \\
\hline & Saliva & $\mathrm{P}$ & 29.15 & 3 \\
\hline \multirow{2}{*}{8} & cONS & $\mathrm{P}$ & 29.60 & 4 \\
\hline & Saliva & $\mathrm{P}$ & 26.79 & 6 \\
\hline \multirow{2}{*}{9} & cONS & $\mathrm{P}$ & 26.13 & 6 \\
\hline & Saliva & $\mathrm{P}$ & 22.73 & 8 \\
\hline \multirow{2}{*}{10} & cONS & $\mathrm{P}$ & 23.45 & 0 \\
\hline & Saliva & $\mathrm{N}$ & & 3 \\
\hline \multirow{2}{*}{11} & cONS & $\mathrm{P}$ & 29.89 & 5 \\
\hline & Saliva & $\mathrm{P}$ & 31.92 & 8 \\
\hline \multirow{2}{*}{12} & cONS & $\mathrm{P}$ & 29.73 & 5 \\
\hline & Saliva & $\mathrm{N}$ & & 8 \\
\hline \multirow{2}{*}{13} & cONS & $\mathrm{P}$ & 19.24 & 1 \\
\hline & Saliva & $\mathrm{N}$ & & 2 \\
\hline \multirow{2}{*}{14} & cONS & $\mathrm{P}$ & 23.02 & 4 \\
\hline & Saliva & $\mathrm{P}$ & 24.27 & 5 \\
\hline \multirow{2}{*}{15} & cONS & $\mathrm{P}$ & 25.58 & 4 \\
\hline & Saliva & $\mathrm{P}$ & 20.08 & 4 \\
\hline \multirow{2}{*}{16} & cONS & $\mathrm{P}$ & 36.74 & 10 \\
\hline & Saliva & $\mathrm{P}$ & 30.24 & 11 \\
\hline \multirow{2}{*}{17} & cONS & $\mathrm{P}$ & 29.10 & 0 \\
\hline & Saliva & $\mathrm{P}$ & 31.27 & 3 \\
\hline \multirow{2}{*}{18} & cONS & $\mathrm{P}$ & 17.37 & 5 \\
\hline & Saliva & $\mathrm{N}$ & & 6 \\
\hline \multirow{2}{*}{19} & cONS & $\mathrm{P}$ & 31.21 & 7 \\
\hline & Saliva & $\mathrm{N}$ & & 8 \\
\hline \multirow{2}{*}{20} & cONS & $\mathrm{P}$ & 32.91 & 8 \\
\hline & Saliva & $\mathrm{P}$ & 21.18 & 10 \\
\hline
\end{tabular}

cONS: combined oropharyngeal and nasopharyngeal swab, $N$ : Negative, $P$ : Positive $^{*}: p>0.05$

We compared the diagnostic validity of saliva samples (n: 20) collected prospectively from patients whose cONS samples were tested positive. In 12 (60\%) patients positivity was detected in both samples. Detection time for SARS CoV-2 RNA positivity in saliva after first clinical symptom varied from one to twelve days. The median Ct value was 25.50 (IQR: 17.37-36.74) in cONS samples and 28.00 (IQR: 20.08-31.00) in saliva samples (Table VI). Cycle threshold values detected from cONS samples were lower than saliva samples, the difference was not found statistically significant ( $p>0.05)$. We also added 12 saliva samples from patients whose
cONS samples were tested negative for SARS CoV-2 RNA despite strong clinical suspicion. Surprisingly, in $4(30 \%)$ patients saliva samples were found to be positive (data not shown) even after 20 days from the symptom onset.

\section{DISCUSSION}

In this study, we found that the most common symptom was cough (66.22\%), followed by dyspnea (31.69\%) and fever (31.66\%) in patients with suspected COVID-19 infection (Table I). SARS CoV-2 RNA has been investigated in cONS samples (n: 5819), sputum (n: 39), and TA (n: 34) samples from 4812 patients with suspected COVID-19 infection. Retrospective analysis of our patients revealed a $19.66 \%$ positivity rate for cONS samples (1144 of 5819), 29.41\% for TA samples (10 of 34), and $30.77 \%$ for sputum samples (12 of 39) (Table II).

The course of COVID-19 varies among individuals ranging from asymptomatic infection to severe respiratory failure [16]. Common symptoms of the disease are fever, cough, slight dyspnea, sore throat, fatigue and headache, therefore, it is difficult to differentiate COVID-19 from other respiratory diseases [17]. According to the WHO interim guideline, the primary and preferred method for diagnosis is the collection of upper respiratory tract samples via nasopharyngeal and oropharyngeal swabs [2]. The use of bronchoscopy is not recommended as the aerosol that is generated poses a substantial risk for both patients and healthcare staff and tracheal aspiration can be used to collect respiratory specimens in intubated patients [18]. One of the earliest papers about laboratory diagnosis published in February 2020 compared the positive ratio of SARS CoV-2 nucleic acid amplification test results from different samples including oropharyngeal swab, blood, urine, and stool of 19 patients. The positive ratio of nucleic acid detection was only $47.4 \%$ in oropharyngeal swab samples, with no positivity in the blood and urine samples [7]. Testing of respiratory samples from multiple sites seems to improve the sensitivity and reduce false-negative test results. Wang et al., reported that bronchoalveolar lavage fluid specimens showed the highest positive rates (14 of $15 ; 93 \%)$, followed by sputum (72 of $104 ; 72 \%$ ), nasal swabs ( 5 of $8 ; 63 \%$ ), and pharyngeal swabs (126 of 398; 32\%) [19]. Lui et al., reported a $38.25 \%$ positivity rate of nasal, and pharyngeal swab samples (n: 4818 ) and $49.12 \%$ positivity of sputum (n: 57 ) samples [20].

During the pandemic, about 40,000 RT-PCR tests have been performed on a daily basis in Turkey and a test kit, validated by The Turkish Ministry of Health, General Directorate of Public Health, Department of Microbiology Reference Laboratories and Biological Products (HSGM), was distributed to 129 authorized diagnostic laboratories. Viral RNA-based tests are the best option to diagnose an acute illness. The true clinical sensitivity of PCR tests is unknown. It is important to remember that the accuracy of the test is affected by the quality of the sample, days from symptom onset to test (very early or late in the infection), technical reasons (virus mutation or PCR inhibition), and whom to test.

In this study, we detected lower Ct values of cONS samples than sputum and TA samples, indicating higher viral loads (Table II). 
The first study to analyze serial samples (throat swabs, sputum, urine, and stool) from two patients in Beijing revealed that the viral loads in throat swab and sputum samples peaked at around 5-6 days after symptom onset, ranging from around 10, 000 to $10,000,000$ copies/mL during this time [10]. A recent and very comprehensive study estimated the viral loads in more than 3000 samples collected from 96 patients analyzed the temporal change in viral loads and the correlation between viral loads in different sample types and disease severity. In the respiratory samples, the median duration of the virus in patients with severe disease (21 days, 14-30 days) was significantly longer than in patients with mild disease ( 14 days, $10-21$ days; $\mathrm{P}=0.04$ ). Patients with severe disease had significantly higher viral loads than patients with mild disease. In the mild group, the viral load was greater during the initial stages of the disease, reached a peak in the second week from disease onset, and was followed by lower loads. In the severe group, however, the viral load in respiratory samples continued to be high during the third and fourth weeks after disease onset [21]. In the majority of our patients (86.72\%) SARS CoV-2 RNA was detected in the first cNOS sample whereas in only $13.28 \%$ of the patients' positive results were obtained in consecutively taken samples within 9 days and only $0.19 \%$ of the patients $4^{\text {th }}$ sample was required for positivity (Table III). Repetitive specimens should be tested in patients with negative RT-PCR and high suspicion or probability of infection to reduce false-negative results. Li et al., reported a potentially high false-negative rate of RT-PCR testing for SARS CoV-2 in the 610 hospitalized patients from whom 241(39.5\%) patients were finally confirmed with COVID-19 with at least one positive RT-PCR test result [22]. Among the 384 patients with initial negative results, the second test was performed and the test results were positive in 48 cases $(12.5 \%)$, seven patients were eventually confirmed with COVID-19 by three repeated swab PCR tests, four were confirmed by four repeated tests, and one was confirmed by five repeated tests. In another reported case, the third time RT-PCR test result for pharyngeal swab specimen from an infected patient turned to be positive after two previous negative results of the PCR test.

We also analyzed consecutive cONS and sputum samples or TA samples from 52 patients. Six lower respiratory tract samples (4 sputum, 2 TA) were found to be positive while cONS samples were found negative (Table IV). Therefore, if a negative result is obtained from a patient with a high suspicion for COVID-19, if possible the lower respiratory tract specimens should be tested. In 7 of 11 patients' clinical condition was severe requiring oxygen support or admission to the intensive care unit. Detection time for SARS CoV-2 RNA in lower respiratory specimens after symptom onset varied 3 to 12 days. The median $\mathrm{Ct}$ value was 30.00 (IQR: 25.85-39.92) in sputum and TA samples and 25.00 (IQR: 18.01-26.39) in cONS samples. Cycle threshold values detected from cONS samples were significantly lower than sputum and TA samples $(\mathrm{p}<0.05)$. Lower $\mathrm{Ct}$ values detected in cONS samples compared to lower respiratory tract specimens were inconsistent with the findings of the previous studies $[19,23]$. We assume that this might be related to the manual extraction method and the performance of our RT-PCR kit for lower respiratory tract samples.
Additionally, we investigated the diagnostic value of saliva as a non-invasive specimen in a small group of hospitalized patients. SARS CoV-2 RNA was detected in 12 of $20(60 \%)$ cONS positive and 4 of $12(30 \%)$ cONS negative patients. Median Ct value was 25.50 (IQR: 17.37-36.74) in cONS samples and 28.00 (IQR: 20.08-31.00) in saliva samples indicating cONS contains a higher viral load. Similar to our results, the median Ct value was found to be lower in NPS compared to saliva in the study performed by Williams et al. and McCormick-Baw et al. [13, 24]. Saliva samples were found to be positive in 4 (30\%) of 12 patients whose cONS samples were tested negative (data not shown). Ct values of four saliva samples were 28.54, 29.89, 29.90 and 31.27 (Median: 29.89, IQR: 28.54-31.27). Previous studies have reported that positive salivary results can be detected from the patients even when their pharyngeal swabs tested negative [13, 24-26]. In our study saliva was found to be positive even after 20 days of the initial symptoms that might alert people about the possibility of transmission through their saliva by close contact.

In a conclusion, the efficacy of the PCR test in the diagnosis of COVID-19 infection is greatly dependent on the pre-analytical phase including patient selection, material collection, and extraction method of RNA and performance of the RT-PCR test kit.

In this study, the preliminary findings of the diagnostic value of saliva for COVID-19 infection also were shared. Further research with larger sample size on saliva efficacy for detecting COVID-19 is needed for confirmation. Although, NPS samples show greatest diagnostic guidance, saliva testing might be an alternative screening test in the face of shortages of swabs and personal protective equipment.

\section{Compliance with Ethical Standards}

\section{Ethical approvals}

The Turkish Ministry of Health Approval: The study protocol was approved by the Turkish Ministry of Health (Protocol Number:2020-05-05T12_38_13).

Institutional Approval: The study protocol was approved by the Institutional Review Board and Ethics Committee of Marmara University, Faculty of Medicine (Protocol Number: 09.2020.563).

Financial Support: The authors have no relevant financial information to disclose.

Conflict of Interest: The authors have no potential conflicts to disclose.

Author contributions: R.C.S.: Method determination, doing PCR test, analyse PCR results, manuscript preparation, D.G.: Doing PCR test, analyse PCR results, manuscript preparation, B.E.S.: Patient selection, sample collection, medical record collection, V.K. : Patient selection, manuscript preparation, A.K.: Method determination, patient selection, manuscript preparation. All authors have read and approved the final version of the article. 


\section{REFERENCES}

[1] Zhu N, Zhang D, Wang W et al. China Novel Coronavirus Investigating and Research Team. A Novel coronavirus from patients with pneumonia in China, 2019. N Engl J Med 2020;382:727-33. doi:10.1056/NEJMoa2001017.

[2] World Health Organization. Diagnostic testing for SARSCoV-2, interim guidance. https://www.who.int/publications/i/ item/diagnostic-testing-for-sars-cov-2 (accessed 20 September 2020).

[3] WorldHealthOrganization.COVID19WeeklyEpidemiological Update, file://C:/Users/HP/Downloads/20201201_Weekly_ Epi_Update_16.pdf, (accessed 3 December 2020).

[4] Turkish Ministry of Health, https://covid19.saglik.gov.tr/, (accessed 3 December 2020).

[5] See A, Toh ST. Respiratory sampling for severe acute respiratory syndrome coronavirus 2: An Overview. Head Neck 2020;42:1652-6. doi:10.1002/hed.26232.

[6] Zou L, Ruan F, Huang M, et al. SARS-CoV-2 Viral load in upper respiratory specimens of infected patients. $\mathrm{N}$ Engl J Med 2020;382:1177-9. doi: 10.1056/NEJMc2001737.

[7] Xie C, Jiang L, Huang G, et al. Comparison of different samples for 2019 novel coronavirus detection by nucleic acid amplification tests. Int J Infect Dis 2020;93:264-7. doi: 10.1016/j.ijid.2020.02.050.

[8] Centers for Disease Control and Prevention. Interim Guidelines for Collecting. Handling, and Testing Clinical Specimens from Persons for Coronavirus Disease 2019 (COVID-19). https://www.cdc.gov/coronavirus/2019-ncov/ lab/guidelines-clinical-specimens.html, (accessed September 19, 2020).

[9] The Republic of Turkey, Ministry of Health, Directorate General of Public Health, COVID 19 (SARS-CoV-2 Infection) Guide, Study of Scientific Board, General Information, Epidemiology and Diagnosis, 30 May 2020, https://hsgm. saglik.gov.tr/depo/birimler/goc_sagligi/covid19/rehber/ COVID-19_Rehberi20200414_eng_v4_002_14.05.2020.pdf, (accessed 1 November 2020).

[10] Pan Y, Zhang D, Yang P, Poon LLM, Wang Q. Viral load of SARS-CoV-2 in clinical samples. Lancet Infect Dis 2020;20:411-2. doi:10.1016/S1473-3099(20)30113-4.

[11] Huang Y, Chen S, Yang Z, et al. SARS-CoV-2 viralload in clinical samples from critically ill patients. Am J Respir Crit Care Med 2020;201:1435-8. doi:10.1164/rccm.202.003.0572LE.

[12] Patel R, Babady E, Theel ES, et al. Report from the American Society for Microbiology COVID-19 International Summit, 23 March 2020: Value of diagnostic testing for SARS-CoV-2/ COVID-19. mBio 2020;11:e00722-20. doi: 10.1128/mBio.

[13] Williams E, Bond K, Zhang B, Putland M, Williamson DA. Saliva as a noninvasive specimen for detection of SARSCoV-2. J Clin Microbiol 2020;58:e00776-20. doi: 10.1128/ JCM.00776-20.

[14] To KK, Tsang OT, Leung WS, et al. Temporal profiles of viral load in posterior oropharyngeal saliva samples and serum antibody responses during infection by SARS-CoV-2: an observational cohort study. Lancet Infect Dis 2020;20:575-84. doi:https://doi.org/10.1016/S1473-3099(19)30626-7.

[15] Guidance on regulations for the transport of infectious substances 2019-2020. Geneva: World Health Organization: https://www.who.int/ihr/publications /WHO - WHECPI-2019.20/en/. (accessed 10 September 2020).

[16] Yang J, Zheng Y, Gou X, et al. Prevalence of comorbidities and its effects in patients infected with SARS-CoV-2: a systematic review and meta-analysis. Int J Infect Dis 2020;94:91-5. doi: 10.1016/j.ijid.2020.03.017.

[17] Lauer SA, Grantz KH, Bi Q, et al. The incubation period of coronavirus disease 2019 (COVID-19) from publicly reported confirmed cases: Estimation and application. Ann Intern Med 2020;172:577-82. doi: 10.7326/M20-0504.

[18] Pascarella G, Strumia A, Piliego C, et al. COVID-19 diagnosis and management: a comprehensive review. J Intern Med 2020;288:192-206. doi: 10.1111/joim.13091.

[19] Wang W, Xu Y, Gao R, et al. Detection of SARS-CoV-2 in different types of clinical specimens. JAMA 2020;323:1843-4. doi:10.1001/jama.2020.3786.

[20] Liu R, Han H, Liu F, et al. Positive rate of RT-PCR detection of SARS-CoV-2 infection in 4880 cases from one hospital in Wuhan, China, from Jan to Feb 2020. Clin Chim Acta 2020;505:172-5. doi:10.1016/j.cca.2020.03.009.

[21] Zheng S, Fan J, Yu F, et al. Viral load dynamics and disease severity in patients infected with SARS-CoV-2 in Zhejiang province, China, January-March 2020: retrospective cohort study. BMJ 2020;369:m1443. doi: 10.1136/bmj.m1443.

[22] Li Y, Yao L, Li J, et al. Stability issues of RT-PCR testing of SARS-CoV-2 for hospitalized patients clinically diagnosed with COVID-19. J Med Virol 2020;92:903-908. doi: 10.1002/ jmv.25786.

[23] Yu F, Yan L, Wang N et al. Quantitative Detection and Viral Load Analysis of SARS-CoV-2 in Infected Patients. Clin Infect Dis. 2020;71:793-798. doi: 10.1093/cid/ciaa345. PMID: 32221523; PMCID: PMC7184442.

[24] McCormick-Baw C, Morgan K, Gaffney D, et al. Saliva as an alternate specimen source for detection of SARS-CoV-2 in symptomatic patients using Cepheid Xpert Xpress SARSCoV-2. J Clin Microbiol 2020;58:e01109-20. doi: 10.1128/ JCM.01109-20.

[25] Azzi L, Carcano G, Gianfagna F, et al. Saliva is a reliable tool to detect SARS-CoV-2. J Infect 2020;81:e45-e50. doi:10.1016/j. jinf.2020.04.005.

[26] Pasomsub E, Watcharananan SP, Boonyawat K, et al. Saliva sample as a non-invasive specimen for the diagnosis of coronavirus disease 2019: a cross-sectional study. Clin Microbiol Infect 2020 :S1198-743X(20)30278-0. doi:10.1016/j.cmi.2020.05.001. 